\title{
Sexualidade feminina na menopausa: um olhar de maior visibilidade
}

\author{
Female sexuality in menopause: a more visible look
}

Sexualidad femenina en menopausia: una mirada de mayor visibilidade

Andreza Nogueira Silva ${ }^{1}$, Alyce Brito Barros ${ }^{1 *}$, lannaele Oliveira do Vale Batista ${ }^{1}$, José Rafael Eduardo Campos', Sabrina Martins Alves'1, Maria Leni Alves Silva', Petrúcya Frazão Lira', Emanuel Cardoso Monte', Willma José de Santana ${ }^{2}$, Dayse Christina Rodrigues Pereira Luz².

\section{RESUMO}

Objetivo: Analisar a percepção das mulheres sobre sexualidade no período da menopausa. Métodos: Trata-se de um estudo a campo do tipo exploratório, descritivo, com abordagem qualiquantitativo. A pesquisa foi realizada em um Centro de Referência do Idoso (CRI), serviço público, localizado no município de Juazeiro do Norte - CE. Participaram da pesquisa mulheres idosas que estejam sendo acompanhada regularmente pelo $\mathrm{CRI}$ e com idade igual ou acima de 60 anos. Para a coleta de dados utilizou-se uma entrevista semiestruturada auto elaborada sobre o tema, realizada no período de 2019.2, contemplando 18 amostras, submetido à técnica de análise de conteúdo de Bardin. Resultados: Com os dados obtidos avaliamos que muitas idosas não reconhecem a menopausa como um processo fisiológico, sendo perceptível que o processo de alterações da menopausa, envelhecimento e a falta de informações tiveram impacto negativo e significante na diminuição da sexualidade na idosa. Conclusão: Dessa forma a sexualidade na menopausa é um assunto importante e íntimo, por isso poucas mulheres estão preparadas para vivenciá-la, entretanto a enfermagem tem diversas possibilidades de realizar ações com este público, objetivando maior visibilidade e promoção de qualidade de vida.

Palavras-chave: Envelhecimento, Menopausa, Sexualidade.

\begin{abstract}
Objective: To analyze women's perception of sexuality during menopause. Methods: This is an exploratory, descriptive, field study with a quantitative quali approach. The research was carried out in a Reference Center for the Elderly (CRI), a public service, located in the municipality of Juazeiro do Norte - CE. Participated in the research elderly women who are being regularly monitored by the $\mathrm{CRI}$ and aged 60 years or over. For data collection, a self-structured semi-structured interview on the topic was used, conducted in the period 2019.2, covering 18 samples, submitted to Bardin's content analysis technique. Results: With the data obtained, we evaluated that many elderly women do not recognize menopause as a physiological process, and it is noticeable that the process of changes in menopause, aging and the lack of information had a negative and significant impact in decreasing sexuality in the elderly. Conclusion: Thus, sexuality in menopause is an important and intimate subject, therefore few women are prepared to experience it, however nursing has several possibilities to carry out actions with this public, aiming at greater visibility and promotion of quality of life.
\end{abstract}

Keywords: Ageing, Menopause, Sexuality.

\section{RESUMEN}

Objetivo: Analizar la percepción de las mujeres sobre la sexualidad durante la menopausia. Métodos: Este es un estudio exploratorio, descriptivo, de campo con un enfoque calidad cuantitativa. La investigación se

${ }^{1}$ Faculdade de Juazeiro do Norte (FJN), Juazeiro do Norte - CE. *E-mail: alyce.brito@hotmail.com

SUBMETIDO EM: 4/2020

ACEITO EM: 5/2020

PUBLICADO EM: 7/2020

REAS/EJCH | Vol.Sup.n.51 | e3413 | DOI: https://doi.org/10.25248/reas.e3413.2020 Página 1 de 10 
llevó a cabo en un Centro de Referencia para Personas Mayores (CRI), un servicio público, ubicado en el municipio de Juazeiro do Norte - CE. Participó en la investigación de mujeres mayores que están siendo monitoreadas regularmente por el IRC y de 60 años o más. Para la recopilación de datos, se utilizó una entrevista semiestructurada autoestructurada sobre el tema, realizada en el período 2019.2, que abarca 18 muestras, sometidas a la técnica de análisis de contenido de Bardin. Resultados: Con los datos obtenidos, evaluamos que muchas mujeres de edad avanzada no reconocen la menopausia como un proceso fisiológico, y es notable que el proceso de cambios en la menopausia, el envejecimiento y la falta de información tuvieron un impacto negativo y significativo en la disminución de la sexualidad en las personas mayores. Conclusión: Por lo tanto, la sexualidad en la menopausia es un tema importante e íntimo, por lo tanto, pocas mujeres están preparadas para experimentarla, sin embargo, la enfermería tiene varias posibilidades para llevar a cabo acciones con este público, con el objetivo de una mayor visibilidad y promoción de la calidad de vida.

Palabras clave: Envejecimiento, Menopausia, Sexualidad.

\section{INTRODUÇÃO}

Atualmente no Brasil existem cerca de 30 milhões de mulheres entre 45 a 65 anos, ou seja, $32 \%$ da população feminina esta passando pelo período do climatério e a menopausa, com a expansão da expectativa de vida, acredita-se que estas passam um terço da vida na menopausa.

Etapa compreendida como transição do período reprodutivo para não reprodutivo; onde a menopausa é dita como marco na vida da mulher, representando o último fluxo menstrual, através da ausência da menstruação por um período de 12 meses consecutivos (SANTOS JL, et al., 2016; REZENDE FCB, et al., 2019). O Climatério e a menopausa trazem transformações significativas nas dimensões emocionais, sexuais e sociais. A menopausa para a mulher retrata o envelhecimento, vinculando alterações orgânicas e corporais como o fim da sua estética, acompanhado de variáveis graus de desequilíbrio emocionais.

A sexualidade, por exemplo, pode ser vivida nessa fase, na menopausa é entendida numa perspectiva ampla, não se limitando ao ato sexual, mas ao carinho, respeito, sensualidade, zelo, vivida de diferentes formas e de acordo com a cultura do indivíduo. É vista como uma manifestação presente nas fases da vida de um ser humano (MARCEDO RMS, et al., 2017; OLIVEIRA EL, et al., 2018).

Essas mudanças que afligem o psicossocial influenciam a função sexual normal. Algumas mulheres com problemas sexuais como ansiedade, diminuição da lubrificação vaginal, baixo libido sexual, insatisfação entre outros, não procuram a assistência para que obtenham resolução, afirmando que sejam motivos vinculados à: barreiras de opinião, tabus, falta de privacidade e vergonha de falar sobre sexo e menopausa. Sendo que a sexualidade é um dos pilares para a qualidade de vida (BRADAM AV, et al., 2007).

Para os autores Paiva LL e Frasson AL (2014), menopausa e envelhecimento deveriam ser trabalhados em inúmeros aspectos e maneiras, dando visibilidade às modificações do corpo das mulheres e que percebam esses inúmeros sinais, sabendo lidar com mudanças que têm impacto tão grande nas suas vidas.

Compreende-se que a menopausa interfere diretamente na sexualidade da mulher, levando a uma resposta lenta, refletindo na diminuição do ato sexual. A menopausa é vista como prejudicial á saúde da mulher. Acredita-se que a ausência da educação em saúde possa estar contribuindo para a construção de conceitos errôneos sobre sexualidade na pós-menopausa.

A partir do exposto, percebe-se a necessidade de aprofundamento temático, através dos profissionais para um melhor auxílio, assim como, para a quebra do preconceito na comunidade feminina. Surgindo os questionamentos: Por que a menopausa é vista como patologia pelas mulheres? Quais as dificuldades enfrentadas? Por que a sexualidade na menopausa não tem visibilidade?

Tais informações emergem a hipótese de que com o emprego de educações em saúde com o publico alvo, as mulheres em processo de menopausa e pós-menopausa, receberão informações sobre esse 
período, incentivando o reconhecimento das alterações corporais, contribuindo para uma melhor qualidade de vida. O estudo justifica-se por tratar da temática sexualidade na menopausa, pouco discutido e trabalhado na saúde, além de encarada negativamente no público feminino, um momento fisiológico no ciclo vital que traz modificação física, psicológica e comportamental, modificando diretamentea qualidade de vida da mulher.

O estudo auxiliará sensibilização dos acadêmicos e profissionais sobre vivencias e conhecimento das mulheres sobre pós-menopausa. Espera-se que os agentes atuantes trabalhem formulando estratégias para atender às mulheres de forma holística, todas as dimensões.

Por isso, se torna relevante à medida que incentiva disseminação de informação sobre a menopausa e sexualidade, quebrando tabus, sendo coadjuvante para a educação em saúde nessa população carente de conhecimentos sobre a temática. O presente estudo tem como objetivo analisar a percepção das mulheres sobre a sexualidade no período da menopausa.

\section{MÉTODOS}

Trata-se de uma pesquisa exploratória, descritiva, com abordagem qualiquantativa. Realizou-se em um Centro de Idoso em município do Ceará, onde são ofertadas atividades como hidroginástica, alongamento, dança, natação, assistência ocupacional, além de consultas multiprofissionais. A escolha do local foi intencional, acreditando que possuir vínculos com os $\mathrm{Cl}$ e instituição de ensino simboliza uma oportunidade de parcerias com novos projetos a serem desenvolvidos com o público e foco sexualidade na terceira idade.

Constituíram-se como público-alvo idosas, que atendiam aos critérios de inclusão: mulheres que são acompanhadas pelo $\mathrm{Cl}$, frequentadoras uma vez por semana, com idade igual ou acima de sessenta (60) anos, com condições emocionais estáveis para responder o questionário e não estejam realizando tratamento psiquiátrico, assim como a voluntariedade de participação.

O projeto foi submetido à avaliação do Comitê de Ética em Pesquisa da Faculdade de Juazeiro do Norte - CEP/FJN e, mediante parecer favorável de número 3.698.506, foi realizada a coleta dos dados. Os participantes foram previamente informados a respeito dos objetivos e procedimentos da pesquisa, bem como, da individualidade, confiabilidade dos dados e do anonimato da sua colaboração. Foi destacado o caráter voluntário da pesquisa, deixando-os à vontade para escolher participar ou não. Em seguida, foi solicitado que lessem e assinassem o Termo de Consentimento Livre e Esclarecido - TCLE.

$\mathrm{Na}$ coleta de dados utilizou-se a técnica de entrevista aberta e fechada, denominada entrevista semiestruturada combinando perguntas objetivas e subjetivas, onde o informante tem a possibilidade de discorrer sobre o tema proposto. O pesquisador deve seguir um conjunto de questões pré-definidas, fazendo-o em um contexto semelhante ao de uma conversa informal (BONI V e QUARESMA SJ, 2005).

Assim, os participantes responderam questionário auto elaborado, objetivando-se obtenção do perfil da amostra, contendo 30 perguntas de fácil decisão de escolha das alternativas, baseadas em três temas: "dados sociodemográficos", "experiências e conhecimentos" e "a enfermagem na saúde da mulher na menopausa", de forma sistematizada de acordo com os objetivos do estudo.

A entrevista foi preenchida mediante resposta das participantes, que explanaram de forma livre e aberto sobre o significado da sexualidade. Em seguida submetida à análise de dados com o objetivo de agrupar as informações nas categorias temáticas utilizadas na entrevista para discursão. Foram contempladas 18 amostras, na qual o número se deu por saturação, segundo Vieira $S$ (2010), saturação é o ponto da coleta de dados em que nenhuma informação nova surge de uma nova entrevista.

Os dados coletados foram submetidos à análise conteúdo, à técnica de Análise de Conteúdo Temático de acordo com a autora Bardin, que se refere a um conjunto de técnicas de análise das comunicações visando obter indicadores que permitam a inferência de conhecimentos relativos às condições de produção/recepção das mensagens (BARDIN L, 2011).

A análise indica que a utilização da análise de conteúdo baseia-se por três fases fundamentais a 1- préanálise: onde fazemos uma leitura flutuante do material direcionando sua hipótese e objetivos, 
reorganização propriamente dita da entrevista. 2- Exploração do material: administrando as técnicas do corpus; realizando o recorte, categorização e codificação, definição das categorias em eixos temáticos. 3Tratamento dos resultados: Realizando síntese e seleção dos resultados, provas de validação e interpretações das respostas obtidas com a condensação e o destaque das informações.

Esta é a fase da descrição da análise do material coletado em aprofundamento, analisado por referenciais teóricos e discutidos posteriormente, onde ocorrerá a união de pensamentos e a classificação temática, possibilitando a interpretação (BARDIN L, 2011). Para construções de tabelas foi utilizada o Microsoft Word 2016.

\section{RESULTADOS E DISCUSSÃO}

Mediante a análise e síntese do material coletado, estabeleceram-se três eixos interpretativos que subsidiaram nossa discussão, a saber: 3.1 - Dados sociodemográfico das participantes; 3.2 - Experiências e conhecimentos; 3.3 - A enfermagem na saúde da mulher.

\subsection{Dados sociodemográficos das participantes}

Nesta primeira etapa, é apresentado o perfil das participantes da pesquisa (Tabela 1), caracterizando os dados consolidados em sociais e demográficos.

Houve participação de 18 mulheres idosas, das quais 72,2\% tinha idade acima de 77 anos e apenas $11,11 \%$ com idade entre 60 a 64 anos. Destas, $66,66 \%$ considerava-se de raça parda e em religiosidade $100 \%$ católica romana. No que tange estado civil $50 \%$ das participantes estava separada/viúva e apenas $16,67 \%$ encontravam-se casada/união estável e 22,78\% encontrava-se em nenhum relacionamento amoroso. Semelhando-se com estudo realizado por Marcedo RMS (2016) com idosos no município de Barão de Antonina - SP.

Vale ressaltar que a maioria das participantes cerca de $66,66 \%$ eram analfabetas não tendo contanto nenhum com a escola na fase infanto-juvenil e apenas 5,56\% tinha o nível superior. A escolaridade intervém diretamente no desenvolvimento e compreensão de saúde, na habilidade de compreender o tratamento prescrito e na prática do autocuidado, interferindo diretamente na relação paciente/profissional de saúde (MACEDO DC, 2016).

A maioria não possuía atividade renumerada se declarando "do lar", apenas 5,56\% realiza atividade com renumeração, e em cerca de $94,44 \%$ a renda familiar não chega a 2 salários mínimos, além de 16,66\% moram com 1 pessoa ou sozinha, estes dados que se assemelham a um estudo realizado no município de Vitória da Conquista/BA, por Nascimento CP et al. (2015) tornando limitada o acesso de muitos idosos a bens e consumo na sociedade afetando diretamente a qualidade de vida desses idosos em ambos os estudos.

Quanto à saúde e doença, 5,56\% faz uso de álcool e fumo e $66,66 \%$ tem comorbidades associadas como Hipertensão arterial sistêmica - HAS e osteoporose, fazendo uso de medicações em longo prazo. Como ponto positivo $94,44 \%$ realiza atividade física no centro de atendimento onde são cadastradas. Estudos afirmam que a realização de exercícios físicos contribui com a diminuição dos sintomas desenvolvidos pelo climatério e menopausa, tornando-se menos intensos fornecendo para a mulher sensações de bem-estar e consequentemente tem melhor qualidade de vida (SANTOS JL, et al., 2016). 
Tabela 1 - Perfil sociodemográfico.

\begin{tabular}{|c|c|}
\hline Variáveis & Número/Percentual \\
\hline \multicolumn{2}{|l|}{ Idade (em anos) } \\
\hline 60 a 64 & $2(11,11 \%)$ \\
\hline 65 a 69 & $3(16,66 \%)$ \\
\hline$>70$ & $13(72,22 \%)$ \\
\hline \multicolumn{2}{|l|}{ Cor ou raça } \\
\hline Branca & $6(33,33 \%)$ \\
\hline Parda & $12(66,66 \%)$ \\
\hline \multicolumn{2}{|l|}{ Religião } \\
\hline Católica Romana & $18(100 \%)$ \\
\hline \multicolumn{2}{|l|}{ Profissão } \\
\hline Do lar & $17(94,44 \%)$ \\
\hline Fora do lar & $1(5,56 \%)$ \\
\hline \multicolumn{2}{|l|}{ Estado civil } \\
\hline Solteira & $5(22,78 \%)$ \\
\hline Namorando & $1(5,56 \%)$ \\
\hline Casada/união estável & $3(16,67 \%)$ \\
\hline Separada/viúva & $9(50 \%)$ \\
\hline \multicolumn{2}{|l|}{ Escolaridade } \\
\hline Analfabeta & $4(22,22 \%)$ \\
\hline Fund. incompleto & $12(66,66 \%)$ \\
\hline Médio incompleto & $1(5,56 \%)$ \\
\hline Superior & $1(5,56 \%)$ \\
\hline \multicolumn{2}{|l|}{ R. M. (salário mínimo) } \\
\hline 1 & $17(94,44 \%)$ \\
\hline 2 a 3 & $1(5,56 \%)$ \\
\hline \multicolumn{2}{|l|}{ Mora com alguém? } \\
\hline 1 & $3(16,66 \%)$ \\
\hline 2 a 3 & $10(55,55 \%)$ \\
\hline 4 ou + & $2(11,11 \%)$ \\
\hline Sozinha & $3(16,66 \%)$ \\
\hline \multicolumn{2}{|l|}{ Etilista } \\
\hline Sim & $1(5,56 \%)$ \\
\hline Não & $17(94,44 \%)$ \\
\hline \multicolumn{2}{|l|}{ Tabagista } \\
\hline Sim & $1(5,56 \%)$ \\
\hline Não & $17(94,44 \%)$ \\
\hline \multicolumn{2}{|l|}{ Atividade Física } \\
\hline $\operatorname{Sim}$ & $17(94,44 \%)$ \\
\hline Não & $1(5,56 \%)$ \\
\hline \multicolumn{2}{|l|}{ Comorbidades } \\
\hline $\mathrm{DM}$ & $7(38,89 \%)$ \\
\hline HAS & $12(66,66 \%)$ \\
\hline Osteoporose & $12(66,66 \%)$ \\
\hline Outros & $3(16,66 \%)$ \\
\hline \multicolumn{2}{|l|}{ Medicação em uso } \\
\hline Sim & $17(94,44 \%)$ \\
\hline Não & $1(5,56 \%)$ \\
\hline
\end{tabular}

Fonte: Silva AN, et al., 2019. 


\subsection{Experiências e conhecimentos}

As participantes possuem inúmeros conhecimentos acerca da sexualidade e o ser sensual no seu próprio corpo, além das experiências que as perpetuam ao longo de suas vidas.

A menopausa é um momento de transfiguração da mulher, influenciando diretamente na qualidade de vida das mulheres. Pois no que tange as alternações fisiológicas, atinge de forma equânime o estado físico e psíquico da mulher (MELODIE SI e EMILIO HG, 2015; AMARAL ICGA, 2018).

$\mathrm{O}$ estudo demonstra que as mulheres veem a menopausa como algo negativo, associando com a chegada do envelhecimento, doenças e alterações no corpo que atrapalham a rotina, e outras não sabiam sobre termo menopausa, sendo notória a falta de informações, sua fisiologia, sintomas e qualidade de vida durante esse período, vejamos:

“...a menopausa é envelhecimento, só me trouxe coisa ruim, me sinto mal com ela..." (A10)

"A menopausa eu vejo como mudanças no meu corpo, mas não associo isso com saúde, eu não vejo assim." (A11)

"A menopausa é uma fase na vida que todas as mulheres passam por isso, tem que passar." (A15)

Os principais sintomas relacionados à menopausa que foram mencionados pelas participantes foram o calor, irritação, insônia, ansiedade e tristeza. Muitas mulheres relataram sentimentos depressivos com seu corpo, relacionamentos pessoais, associando ao envelhecimento.

"...Sinto muito calor e me irrito com facilidade e por qualquer motivo, odeio quando isso acontece..." (A2)

"Eu sinto muito calor, e às vezes me acho depressiva, com pensamentos negativos..." (A17)

“...irrito-me muito fácil, e tenho muita ansiedade durante todo o meu dia..." (A9)

Nesses depoimentos observamos as mudanças fisiológicas e neuropsíquicas ocorridas durante o climatério e menopausa. Muitas vezes mudanças neuropsíquicas são as primeiras percebidas pelas mulheres (ansiedade, depressão, insônia, tristeza). Essas alterações hormonais têm capacidade de mudarem o cognitivoe isso reflete em insegurança, desconforto nos relacionamentos no cotidiano com o meio social da mulher (REZENDE FCB, et al., 2019). Diante disso, é notória a importância de desenvolverem ações com esse público sobre a temática e como lidar com alterações orgânicas e físicas com mais dignidade.

O climatério e menopausa não são apenas período de transição da reprodução feminina, mas período dotado de mudanças e adaptações no meio biopsicossocial e espiritual. A sexualidade atualmente é voltada para a beleza jovial e não para envelhecimento e sexualidade na mulher idosa rotulando como indesejáveis para $o$ ato sexual. Designando a sexualidade feminina a não liberdade, negando o relacionamento, pois definem o sexo apenas como um ato que envolve genitalidade, não sabendo que o sexo está também conectado ao emocional e afetividade que é algo crucial no ser humano (NETO FAS, et al., 2014; SOUZA $\mathrm{M}$, et al., 2015).

No que tange a sexualidade na menopausa nem todas as mulheres sofrem com alterações da menopausa, mas podem associar os sintomas com o envelhecimento. Porém outros estudos afirmam que a mulher no período de climatério e menopausa, sendo a mesma saudável, não terá interferências em almejar a relação sexual, apenas diminuição da intensidade decorrente da diminuição do hormônio estrogênio, nem por isso a mulher irá perder o entusiasmo e satisfação (FLEURY HJ e ABDO CHN, 2015; OLIVEIRA EL, et al., 2018).

Ao ser debatido sobre sexualidade na menopausa e interferências, as participantes relatam não terem muitas dificuldades durante o sexo, outras apontaram para o desconforto durante a relação, mudanças no 
endotélio e musculatura vaginal decorrentes das alterações hormonais, levando a diminuição da lubrificação vaginal, onde as entrevistadas justificaram o desconforto nisto e que não usavam nada para substituir o lubrificante endógeno.

No período da menopausa e pós-menopausa ocorre mudanças interferindo na sexualidade tais como baixa lubrificação sexual, estreitamento da parede vaginal levando dispareunia. A diminuição dos hormônios estrogênio e progesterona desencadeando modificações nos órgãos genitais internos, interferindo na resposta sexual.

Os sintomas clássicos relacionados com o processo de hipotrofa genital que podem ocorrer devido ao hipoestrogenismo são: ressecamento vaginal, irritação, prurido, e sensação de pressão. Influenciando de forma direta na vida sexual dela, causando dor, insatisfação e ansiedade (Ministério da Saúde, 2016).

"Não percebi muitas mudanças, apena um leve desconforto durante a relação, mas não dou tanta importância para isso, eu mal faço sexo..." (A2)

"Eu não percebi muita coisa." (A15)

A respeito do ato sexual as participantes afirmaram falta de sexo no envelhecimento, menosprezando o corpo como não atraente, e a insatisfação sexual com o companheiro. Foram debatidos a prática sexual, o tipo de atividade sexual e a satisfação sexual (Tabela 2), dados que $77,78 \%$ das participantes não realizam atividades sexuais, sendo estas solteiras/viúvas não demonstrando nenhum interesse em ter novos relacionamentos atualmente. Observamos nas falas a seguir:

"Não quero mais homem para me dar dor de cabeça, estou velha." (A3)

"Depois do meu marido eu não quero ninguém, ele não me tratava bem, então não quero outro para ser igual a ele." (A6).

“..não me acho tão bonita como antes, e nem mais atraente..." (A8)

"...estamos velhos e o desejo foi embora..." (A7)

Tabela 2 - Perfil das variantes sexuais.

\begin{tabular}{cc}
\hline Atividade sexual & Número/Percentual \\
\hline Sem atividade & $14(77,78 \%)$ \\
$2(11,11 \%)$ \\
$2(11,11 \%)$
\end{tabular}

Fonte: Silva AN, et al., 2019.

Observamos que $44,44 \%$ das participantes sentem satisfação com a relação sexual e $22,78 \%$ mesmo casadas, são insatisfeitas com a relação sexual, durante a entrevista observou-se necessidade de falarem do relacionamento com o companheiro e como sentiam-se sobre.

Muitas falaram que durante o casamento - para muitas era o único -, sentiam falta de carinho, respeito, amor do companheiro, com isso o desejo sexual para elas quase não tinha, pois precisavam se sentir amadas por eles, para outras associavam falta de libido com envelhecimento. Mulheres sexualmente insatisfeitas ativam pensamentos negativos quanto aos companheiros sexuais, aumentando $o$ afastamento e fracasso sexual (FLEURY HJ e ABDO CHN, 2018), vejamos: 
“...não me sinto atraída, me sinto fria, e não estou satisfeita com o sexo com meu marido, mal fazemos..." (A4)

"Eu nunca me sentir amada por ele, ele é muito ignorante, bruto demais, não tenho desejo assim." (A18)

"Eu tento namorar, mas não me sinto atraída, com a velhice isso diminui demais." (A10)

Quanto ao tipo de atividade sexual que as idosas realizam, 94,44\% era por penetração, não realizando outra atividade com companheiro para que ocorra variações de atividades e diminua a monotonia da relação. Também relataram constrangimento em falar sobre sexo com seus parceiros, além de não pesquisarem sobre o assunto. É evidente a falta de comunicação entre o casal para falarem sobre sexo, não compartilhando sentimentos, carinhos e entrega um do outro, até mesmo na juventude onde não falavam sobre $o$ assunto no meio familiar.

A sexualidade envolve uma ampla relação pessoal e interpessoal, ligada não somente na dimensão biológica, mas envolvendo o biopsicossocial e espiritual do homem. Portanto, a sexualidade no envelhecimento envolve sentimentos de amor, respeito, reciprocidade, companheirismo e cuidado. Podendo ser vivida e sentida de várias formas, tais como um momento de atenção, contato físico, proferindo respeito e amor pelo companheiro, momento de autovalorização e afirmação do seu corpo(REZENDE FCB, et al., 2019; SANTOS MV, et al., 2017).

\subsection{Enfermagem na saúde da mulher na menopausa}

A sexualidade nas mulheres é um assunto que circunda em tabus quando discutido socialmente, algo que não é apenas atualmente, vem desde décadas atrás, retratadas como assunto íntimo, onde as mulheres foram educadas em padrões culturais recatados, o sexo era pouco discutido no meio familiar. Estudos ressaltam que esse padrão de educação recatado traz consequências para a vida sentimental e sexual feminina, onde a mesma não foi educada para ser ver, se tocar e se conhecer sexualmente (OLIVEIRA EL, et al., 2018). As participantes relataram dificuldades de comunicação sobre sexualidade quando mencionado sobre métodos realizados para evitar concepção, analisamos nos relatos a seguir:

"... eu nunca ouvir falar sobre isso..." (A3)

“... Se eu soubesse disso, eu não teria tantos filhos, quase todo ano eu estava grávida." (A7)

"... nunca falava sobre sexo, os meus pais quando estavam conversando parava e eu nunca fui informada de nada, simplesmente casei..." (A12)

A falta de informações sobre proteções sexuais, contraceptivos orais e hormonais é algo essencial a ser debatido em relacionamentos pessoais e reprodutivos, muitas participantes cerca de $77,78 \%$ afirmaram nunca fazer uso de contraceptivo, não tinha acesso a informações sobre a existência, uso e acesso aos contraceptivos, apenas $22,22 \%$ relataram que fizeram uso de métodos como dispositivo intrauterino (DIU) e anticonceptivos orais e injetáveis.

Daí, evidenciamos a escassez de ações de enfermagem para a saúde feminina, mesmo sendo uma falha da sociedade, à área da saúde tem parcela de culpa na disseminação de informações. As participantes do estudo relataram que não obteve informações de enfermagem sobre sexualidade, menopausa ou qualquer interferência desenvolvida, apenas informações sobre menopausa em consultas médicas.

As mesmas, participam de atividades coletivas de educação em saúde, porém voltados para diabetes mellitus e HAS. Informações sobre sexualidade na menopausa é um assunto íntimo e escasso na sociedade, não sendo compartilhado informações entre casais, pais, amigos, somente em consultas médicas, onde as mesmas falaram que só conversavam a cerca da menopausa, seus sintomas e a necessidade de tratamento hormonal, onde apenas $22,78 \%$ fazia uso de medicações hormonais para tratamento da menopausa. 
O cuidado de enfermagem é caracterizado pela humanização, percorrendo um olhar integral ao cliente. A enfermagem é capaz de emergir energia e capacidade na vida da idosa, otimizando cuidado e qualidade de vida. O processo de envelhecimento leva a problemas de saúde, e a sexualidade da mulher acaba não sendo observada, porém faz parte das necessidades fisiológicas do ser humano, permanecendo como um grande eixo para o cuidar (TEXEIRA MM, et al., 2012).

Com isso, é evidente influencia da educação e acesso as informações sobre a sexualidade e todas as mudanças que circulam ns menopausa, pesquisas epidemiológicas afirmam que mulheres que têm acesso a essas informações conseguem lidar melhor com as alterações que ocorrem com o próprio corpo, buscando a melhor forma de viver com mais qualidade (CREMA IL e TILIO R, 2017).

Portanto, a consulta de enfermagem com a mulher na menopausa e pós-menopausa deve conter informações de esclarecimento e estímulos. Elucidando sobre a menopausa, mudanças físicas, orgânicas, e reposição hormonal. Sobre anatomia e fisiologia sexual, atividades que podem realizar e substâncias que podem ser utilizadas para melhorar dinâmica sexual. Além de orientações como reforço para mudanças de hábitos como: a importância de hábitos alimentares saudáveis e uma rotina de exercícios físicos, atuando na diminuição do estresse e da depressão, melhorando a função cardiorrespiratória, e aumento da resistência, trazendo sensação de bem-estar, além de trabalhar de forma atuante no relacionamento familiar (Ministério da Saúde, 2016).

Todavia, a equipe de enfermagem ter preparo para trabalhar com essa população, reflitindo respeito, dedicação, compreensão e disponibilidade para dialogar sobre a temática com as mulheres, buscando compreender junto a elas as transformações da menopausa, incentiva-las a construírem estratégias que melhorem a qualidade de vida (ALVES WRP, et al., 2015; FREIRE AM, et al., 2016).

\section{CONCLUSÃO}

Sexualidade na menopausa é ainda pouco debatido na sociedade, observamos preconceito quando se retrata de sexo na mulher idosa. O enfermeiro tem diversas ações para serem realizadas na assistência a idosa, desde a descoberta do corpo da mulher até mesmo ao enfrentamento de obstáculos sexuais, objetivando promover maior qualidade da assistência e na vida desta. Com isto, há careça de maior visibilidade do assunto, novas pesquisas precisam ser desenvolvidas com esta população, estudos que tragam ações educativas e planejamentos de assistência para a mulher idosa e família, além da necessidade de serem trabalhadas ainda no adulto jovem para que com a chegada do envelhecimento essa cliente consiga se adaptar com as mudanças orgânicas que ocorrem, obtendo um bom envelhecimento.

\section{REFERÊNCIAS}

1. AMARAL ICGA. Conhecimento sobre a menopausa de acordo com mulheres brasileiras de meia-idade: um estudo de base populacional. Repositório da Produção Científica e Intelectual da UNICAMP, 2018; [s.n.]

2. ALVES ERP, et al. Climatério: a intensidade dos sintomas e o desempenho sexual. Texto Contexto Enferm, Florianópolis, 2015; 24(1): 64-71.

3. BRADAM AV, et al. Aspectos da sexualidade na menopausa. Arquivos Médicos da Faculdade de Ciências Médicas da Santa Casa de São Paulo, 2007; 52(2): 39-43.

4. BONI V, QUARESMA SJ. Aprendendo a entrevistar: como fazer entrevistas em ciências sociais. Em Tese, 2005; 2(1):68-80.

5. BARDIN L. Análise de conteúdo. Lisboa: Edições 70 Persona, 2011. 229p.

6. Brasil. Ministério da Saúde. Protocolo de atenção básica: saúde da mulher. Instituto Sírio-Libanês de Ensino e Pesquisa, 2016. 230p.

7. Conselho Nacional de Saúde. Resolução N.512 de 07 de abril de 2016, Pub. L. No. 512 (2016).

8. CREMA IL, TILIO R, CAMPOS MTA. Repercussões da Menopausa para a Sexualidade de Idosas: Revisão Integrativa da Literatura. Psicologia: Ciência e Profissão, 2017; 37(3):753-769.

9. FLEURY HJ, ABDO CHN. Sexualidade na menopausa. Diagn Tratamento, 2015; 20(3):117-20.

10. FLEURY HJ, ABDO CHN. Excitação sexual feminina subjetiva. Diagn Tratamento, 2018; 23(2):66-9.

11. FREIRE AL, et al. Assistência de enfermagem à mulher no climatério e sua sexualidade: relato de experiência na atenção básica. Revista eletrônica de trabalhos acadêmicos - UNIVERSO, 2016; 1(1). 
12. MARCEDO RMS, et al. Interfaces entre a menopausa e a sexualidade em idosas: uma revisão sistemática. CIEH Congresso Internacional Envelhecimento Humano, 2017.

13. MACEDO DC. Avaliação da qualidade de vida de idosos participantes e não participantes de grupo de convívio [dissertação]. São Paulo: Universidade Estadual Paulista (UNESP), 2016.

14. MELODIE SI, EMILIO HG. Climaterio y Sexualidad. Rev. Enfermaría Global, 2015; 14(40): 76-85.

15. NASCIMENTO CP, et al. Desempenho motor em idosos participantes de grupos de convivência. Revista Pesquisa em Fisioterapia, 2015; 5(2): 93-101.

16. OLIVEIRA EL, NEVES ALM, SILVA IR. Sentidos de sexualidade entre mulheres idosas: relações de gênero, ideologias mecanicistas e subversão. Psicologia \& Sociedade, 2018; 30: 1-10.

17. OLIVEIRA DM. JESUS MCP, MERIGHI MAB. Climatério e sexualidade: a compreensão dessa interface por mulheres assistidas em grupo. Texto Contexto Enferm, 2008; 17(3): 519-26.

18. OLIVEIRA EL, NEVES ALM, SILVA IR. Sentidos de sexualidade entre mulheres idosas: relações de gênero, ideologias mecanicistas e subversão. Psicologia \& Sociedade, 2018; 30: e166019.

19. PAIVA LL, FRASSON AL. Reflexões sobre menopausa, incontinência urinária, sexualidade e envelhecimento. Estud. Interndiscipl. Envelhec, 2014; 19(3):743- 757.

20. REZENDE FCB, et al. A sexualidade da mulher no climatério. Revista da Universidade Vale do Rio Verde, 2019; 17(1): $1-10$.

21. SANTOS JL, LEÃO APF, GARDENGHI G. Disfunções sexuais no climatério. Reprodução \& Climatério, 2016; 31(2):86-92.

22. NETO FAS, et al. Sexualidade na terceira idade: compreensão e percepção do idoso, família e sociedade. Revista da Universidade Vale do Rio Verde, 2014; 12(1):317-326.

23. SOUZA M, et al. A vivência da sexualidade por idosas viúvas e suas percepções quanto à opinião dos familiares a respeito. Saúde Soc, 2015; 24(3):936-944.

24. SANTOS MC, et al. Percepções e vivências de idosos sobre sua sexualidade. Almanaque Multidisciplinar em Pesquisa, 2017; 1(1): 25-36.

25. SANTOS EI, et al. Atuação do enfermeiro na promoção da qualidade de vida na terceira idade segundo produções científicas brasileiras. Revista Augustus, 2013. 18(35):51 - 62 .

26. TEXEIRA MM, et al. O enfermeiro frente à sexualidade na terceira idade. Revista da Universidade Ibirapuera, 2012; 3: 50-53.

27. VIEIRA S. O tamanho da amostra nas entrevistas qualitativas. Rene, 2010; 11(4):163-173. 